\title{
Sample familiarity and delayed matching in monkeys*
}

\author{
M. R. D'AMATO \\ Rutgers University, New Brunswick, N.J. 08903
}

Two capuchin monkeys were trained on six two-sample delayed matching-to-sample problems, with the delay interval reaching 24 sec. Although one stimulus (green) was common to all six problems, it was no more effective as a sample than the other six (form) stimuli with which Ss had far less experience. Test trials in which a new sample was formed by the superimposition of two of the six form stimuli (1) showed that Ss had discriminated among the six forms, (2) rendered unlikely the possibility that Ss' matching behavior was controlled by overt mediating responses, and (3) provided no evidence that delay gradients are generated primarily by proactive interference.

In delayed matching-to-sample (DMTS), $\mathrm{S}$ is presented with a sample stimulus, and after an interval of time (the delay or retention interval), the sample stimulus reappears along with one or more comparison stimuli. The task of $S$ is to identify the sample, and presumably S's performance reflects the degree of its retention of the sample stimulus. The present study investigated whether or not differential experience with the stimuli serving as samples is an important variable in DMTS. The results of a previous experiment (Etkin \& D'Amato, 1969) suggested that differential experience in the ratio of 3 to 1 did not affect DMTS performance. In the present study, this ratio was increased to 6 to 1 .

\section{SUBJECTS AND APPARATUS}

The two Ss were adult female Cebus apella with previous experimental experience, but not with DMTS. They began each session 18 to $24 \mathrm{~h}$ food deprived. The apparatus was the same as that employed in previous experiments (e.g., D'Amato \& O'Neill, 1971). In brief, the stimuli were provided by five inline stimulus projectors centered at the four corners and midpoint of a $12-\mathrm{cm}$ square, Each of the five projectors was fitted with a transparent plastic key which served as the response mechanism. Depression of a microswitch centered below the five projectors initiated each trial. As projected, all stimulus patterns were composed of white forms on black backgrounds. A green field, which illuminated a circular area $25 \mathrm{~mm}$ in diam, was also used.

\section{PROCEDURE}

To initiate a DMTS trial, $\mathbf{S}$ pressed the microswitch 15 times, upon which

*This research was supported by National Science Foundation Grant GB-24386X. Reprints may be obtained from $\mathrm{Dr} M, R$. D'Amato, Department of Psychology, Rutgers University, 88 College Avenue, New Brunswick. N.J. 08903 . the sample was presented on the center projector. When $S$ pressed the key on the center projector (for a minimum of $0.3 \mathrm{sec}$ ), the sample disappeared and the delay interval began. Near-total darkness prevailed during the delay interval (cf. D'Amato $\&$ O'Neill, 1971). At the end of the delay interval, the sample and the comparison stimulus appeared on two of the outer projectors, chosen quasirandomly. A correct response produced a $190-\mathrm{mg}$ CIBA banana-flavored pellet; an incorrect response was followed by a 1-min time-out signaled by the dimming of the overhead houselight.

There were six DMTS problems, each involving only two stimuli. A green field served as one stimulus in each of the six problems, while the

Table 1 Days to Reach Criterion of $89 \%$ Correct Responses

\begin{tabular}{|c|c|c|c|c|c|c|}
\hline \multirow[b]{2}{*}{$\begin{array}{l}\text { Delay } \\
\text { (Sec) }\end{array}$} & \multicolumn{6}{|c|}{$\begin{array}{l}\text { Problems } \\
\text { Green and: }\end{array}$} \\
\hline & Dot & Circle & Triangle & $\begin{array}{l}\text { Vertical } \\
\text { Line }\end{array}$ & Square & $\begin{array}{l}\text { Horizontal } \\
\text { Line }\end{array}$ \\
\hline 0 & $18(10)^{*}$ & $7(3)$ & $3(5)$ & $2(1)$ & $1(5)$ & $1(1)$ \\
\hline 1 & $3(1)$ & $1(1)$ & $2(1)$ & 1 (1) & $2(2)$ & $1(1)$ \\
\hline 2 & $5(7)$ & $5(3)$ & $2(2)$ & 1 (1) & $1(1)$ & $1(2)$ \\
\hline 3 & $1(1)$ & $1(1)$ & $1(1)$ & 1 (1) & 3 (1) & $2(1)$ \\
\hline 4 & 1 (1) & $1(5)$ & $1(2)$ & $1(7)$ & $1(4)$ & $1(2)$ \\
\hline 6 & $1(1)$ & $1(2)$ & $1(10)$ & $1(2)$ & $2(1)$ & $1+$ \\
\hline 8 & 1 (1) & $1(1)$ & $1(2)$ & $1(1)$ & $2(1)$ & $1(1)$ \\
\hline 10 & $1(1)$ & $3(1)$ & $2(3)$ & 1 (1) & $1(2)$ & 1 (1) \\
\hline 12 & 1 (1) & $1(1)$ & $1(1)$ & 1 (1) & $1(1)$ & $1(1)$ \\
\hline 14 & 1 (1) & $2(3)$ & $2(1)$ & $1(1)$ & 1 (1) & $1(1)$ \\
\hline 16 & $1(1)$ & 1 (1) & 1 (1) & 2 (1) & $2(1)$ & 1 (1) \\
\hline 18 & 1 (1) & $2(1)$ & $2(1)$ & $1(1)$ & $6(1)$ & $1(1)$ \\
\hline 20 & 1 (1) & 1 (1) & 1 (1) & 1 (1) & $1(1)$ & 1 (1) \\
\hline 22 & 1 (1) & 1 (1) & $1(1)$ & $1(1)$ & 1 (1) & $1(1)$ \\
\hline 24 & $1(1)$ & $1(1)$ & $1(1)$ & $1(1)$ & 1 (1) & $1(1)$ \\
\hline$\Sigma$ Days & $38(30)$ & $29(26)$ & $22(33)$ & $17(22)$ & $26(24)$ & $16(16)$ \\
\hline 24 & $96(97)$ & \multicolumn{4}{|c|}{ Percent Correct ( 72 Trials) } & $99(100)$ \\
\hline \multicolumn{7}{|c|}{ Percent Correct With + Replacing Green (36 Trials) } \\
\hline
\end{tabular}

*Dagwood's data shown within parentheses; Fifi's data shown outside. +Not run at this delay. shifted to the next problem, the delay interval remaining at 0 sec. When criterion was achieved on all six problems, the delay interval was increased by $1 \mathrm{sec}$. When Ss reached criterion at the 4-sec delay interval, the latter was advanced in steps of 2 sec until criterion on all six problems was achieved at 24 sec. Each $S$ went through the six problems in a constant, though somewhat different, order.

The Ss were then cycled through the six problems twice (for a total of 72 trials on each problem) to obtain a performance baseline on each task. At this point, the green field was replaced by a stimulus constructed by superimposing the vertical and horizontal lines to form a "+." Still at the 24-sec delay interval, Ss were given

second stimulus was a square for one problem, a circle for another, and a triangle, a vertical line, a horizontal line, or a dot for the remaining four of the six problems. Thus the green field served as sample and as comparison stimulus six times more often than any of the "form" stimuli. Moreover, since the green field was projected on the entire response key and, therefore, presumably required less in the way of "receptor-orienting" responses, it was favored by this factor also.

The design of the experiment is summarized in Table 1. Throughout the major portion of the experiment, Ss were given 36 trials per day until they achieved a criterion of 32 out of 36 correct responses. Training began at 0 -sec delay, and after reaching criterion on one problem, $S$ was

.


one session of 36 trials on each of the six problems, with the + serving in place of the green field.

RESULTS AND DISCUSSION

The results are summarized in Table 1, from which it may be seen that both $\mathrm{Ss}$ eventually became very proficient at delayed matching, showing little decrement in performance as the delay interval was increased up to $24 \mathrm{sec}$. By the end of acquisition training, both $S s$ had received approximately 5,300 trials.

The percentage of correct responses achieved on trials in which the green field served as the sample was calculated for each training day. This figure was compared to the corresponding percentage of correct responses which occurred when the sample stimulus was a form. The difference between these two percentages was then averaged over all training days to yield an estimate of the efficacy of the green field and form stimuli in their roles as samples. The mean difference was only $1 \%$ in the case of Fifi (in favor of the form stimuli) and $1.8 \%$ for Dagwood (in favor of the green field).

Thus, although the green field served as sample (and comparison stimulus) six times more often than any of the form stimuli, it apparently was retained no better than the latter. It appears, therefore, that beyond certain levels of experience, additional exposure to a stimulus does not improve its capability of serving as a sample stimulus in DMTS. Nor, apparently, is the stimulus necessarily stored more efficiently when it occupies a greater part of the response key and thus requires less in the way of receptor orientation. Because of the nature of the dependent variable, however, these conclusions may be open to challenge. Since there was only one comparison stimulus, it is conceivable that $\mathbf{S}$ was more efficient at storing the green field, but this fact was not reflected in differential performance. Such would be the case if Ss were controlled by green and "nongreen" as the sample stimuli rather than by the actual form stimuli employed, a matching strategy which we have observed in a different context (D'Amato \& O'Neill, 1970).

However, the results of the test trials on which + replaced the green field dispute this interpretation. Table 1 (last row) shows that both Ss responded at a high level of accuracy on at least four of the six test problems. Employing the one-tailed binomial test and a .05 level of significance, only one of the test sessions (Fifi on + and triangle) fails to exceed chance expectation. Clearly, these results could not have been obtained if Ss had not discriminated among the six form stimuli during prior acquisition training. It would appear, therefore, that the failure of the green field to emerge as a more effective sample stimulus does not relate to Ss' collapsing the sample set into green and nongreen.

These tests also render unlikely the possibility that overt mediating responses controlled Ss' matching behavior. Both Ss responded with high accuracy at the outset of the test trials long before any distinctive mediating responses could have been conditioned to the + stimulus. Moreover, if the latter stimulus controlled mediating responses by virtue of its component parts (vertical and horizontal lines), such responses would often have been in conflict with the appropriate matching response (as in the + and vertical line problem and the + and horizontal line).

A second purpose behind the substitution of the green field by the + stimulus was to gather information concerning the degree of interference operating in DMTS. A major theoretical task of short-term retention studies is to account for the orderly decrement in performance which occurs as the delay interval is increased, i.e., to account for the delay (retention) gradient. Proactive interference has been frequently cited as a major source of such forgetting (e.g., Jarvik, Goldfarb \& Carley, 1969). In a previous study, we found little evidence to support the view that proactive interference is an important determiner of the delay gradient (Etkin \& D'Amato, 1969), and the results of the test sessions with the + stimulus also seem to point in this direction. Only one of the six problems, + and triangle, suffered a marked decrement in performance. Generally speaking, the performance decrement in those problems where maximum interference would be expected (+ and vertical line, + and square, + and horizontal line) is modest, considering the long delay interval employed and the probability that the introduction of any new stimulus would lead to some performance decrement. Although by no means definitive, the present data, along with those of Etkin and D'Amato (1969), implicate factors other than proactive interference in the generation of the orderly delay gradient s observed in delayed-matching tasks.

\section{REFERENCES}

D'AM ATO, M. R., \& O'NEILL, W. Matching behavior: Some methodological problems. Behavior Research Methods \& Instrumentation, $1970,2,162-164$.

D'AMATO, M. R., \& O'NEILL, W. Effect of delay-interval illumination on matching behavior in the capuchin monkey. Journal of the Experimental Analysis of Behavior, 1971, 15, 327-333.

ETKIN, M., \& D'AMATO, M. R. Delayed matching-to-sample and short-term memory in the capuchin monkey. Joumal of Comparative \& Physiological Psychology, 1969, 69, 544-549.

JARVIK, M. E., GOLDFARB, T. L, \& CARLEY, J. L. Influence of interference on delayed matching in monk eys. Journal of Experimental Psychology, 1969, 81, 1-6. 